\title{
ORIGINAL \\ EVALUATION OF LIVER MASSES ON CT-SCAN
}

PROF-1137

\author{
DR. SYED MEHDI RAZA RIZVI, MBBS, DMRD \\ Associate Professor \& Head, Deptt. of Radiology, \\ Allied Hospital, Faisalabad \\ DR. NASIR IQBAL, MBBS \\ Medical Officer, \\ Deptt. of Radiology, \\ Allied Hospital, Faisalabad
}

\author{
DR. M. AZHAR NASEEM, MBBS, DMRD \\ Radiologist \\ Government General Hospital, \\ Faisalabad
}

Dr. M. Asif Raza, MBBS

Medical Officer

\begin{abstract}
Objective: To evaluate liver masses on CT-Scan. Study design: A retrospective study. Place \& duration of study: This study was carried out in the deptt. of Radiology, Allied Hospital / PMC Faisalabad from 2004 to 2005. Material \& Method: Retrospectively reviewed the case records of 120 patients who underwent CTScan from Jan. 2004 to Dec. 2005. All of the patients had one or more of the signs \& symptoms of jaundice, persistent pain epigastrium, right upper quadrant pain of vague to moderate intensity, weight loss, \& asthenia. Most of them had a mass \& or hepatosplenomegaly on palpation. Result: Ultrasound was used as a screening test for suspicious hepatic tumors. CT was found to be the mainstay and the imaging modality of first choice in the diagnosis of hepatic malignancies. $61.6 \%$ had primary liver neoplasia, $16.7 \%$ had mets from stomach, colorectal, breast, bronchus, ovaries, lymphoma, $8.3 \%$ had liver abscesses, $5 \%$ had focal nodular hyperplasia, $5 \%$ had simple cysts, \& $3.3 \%$ had hemangiomas. Conclusion: Computed Tomography (CT) allows more accurate detection \& characterization of focal hepatic lesions.
\end{abstract}

Key words: Computed Tomography (CT), focal liver masses.

\section{INTRODUCTION}

The common liver masses may be malignant liver tumors like metastatic tumors, hepatocellular carcinoma, \& cholangiocarcinoma. Benign focal liver lesions are cysts, i.e. congenital, parasitic, cavernous hemangiomas, focal nodular hyperplasia, hepatic adenomas, \& abscesses. The useful imaging modalities to investigate liver masses are Computed
Tomography (CT), Ultrasound, \& Magnetic Resonance Imaging (MRI).

Computed Tomography (CT), Delayed CT Arterial Portography (CTAP), Magnetic Resonance Imaging (MRI), Ultrasound \& Intra-operative Ultrasound (IOUS) have all been described as useful pre-operative imaging techniques in patients being considered for 
hepatic resection.

CT-Scan is the best imaging modality to evaluate liver masses. It can identify other intra-abdominal masses \& vascular involvement i.e. thrombosis etc.

\section{MATERIAL \& METHOD}

This study was performed retrospectively on 120 patients having a combination of one or more of the signs \& symptoms of history of jaundice, persistent pain epigastrium, right upper quadrant pain of vague to moderate intensity, weight loss, \& asthenia which were referred from surgical \& medical in-patient wards \& OPD.

Abdominal ultrasound of these patients were performed. Positive reported patients were considered for CT-Scan. All these information were recorded on specially designed proforma.

\section{RESULT}

One hundred \& twenty cases of liver masses were recorded during study period. These patients were referred from the surgical \& medical in-patient wards \& OPD. Age of the patients ranged between 18 to 90 years. 75 patients were male \& 45 patients were female. $72 \%$ of patients had history of jaundice, persistent pain epigastrium, weight loss, asthenia, while rest of the others had vague symptoms with or without hepatosplenomegaly. Underlying history of malignancy other than liver were present in 20 patients who had mets in liver from stomach, colorectal, breast, bronchus, ovaries \& lymphoma. Associated risk factors were smoking, Hepatitis-B \& C infections, cirrhosis \& family history in $50 \%$ cases of all combined malignancies. Ultrasound was used as a screening test for all the suspicious cases of hepatic tumors. CTScans were arranged for all the positively reported cases. On CT-Scan 74 patients had primary liver neoplasm with Male/Female ratio 3:1. 10 patients had abscess in liver. 6 patients had focal nodular hyperplasia. 6 patients had simple cysts. 4 patients had hemangiomas.

\begin{tabular}{|l|c|c|}
\hline Type of liver masses on CT & No of Patient & $\%$ age \\
\hline Hepatocellular carcinoma & $74(56 \mathrm{M}, 18 \mathrm{~F})$ & $61.6 \%$ \\
\hline Mets & $20(6 \mathrm{M}, 14 \mathrm{~F})$ & $16.7 \%$ \\
\hline Liver Abscesses & $10(6 \mathrm{M}, 4 \mathrm{~F})$ & $8.3 \%$ \\
\hline Focal nodular hyperplasia & $6(1 \mathrm{M}, 5 \mathrm{~F})$ & $5 \%$ \\
\hline Simple cysts & $6(4 \mathrm{M}, 2 \mathrm{~F})$ & $5 \%$ \\
\hline Hemangiomas & $4(2 \mathrm{M}, 2 \mathrm{~F})$ & $3.3 \%$ \\
\hline \multicolumn{2}{|c|}{$M=$ Males F= Females } \\
\hline
\end{tabular}

\section{DISCUSSION}

Tumors of the liver may be cystic or solid, benign or malignant. Most are asymptomatic or with vague illdefined symptoms having normal liver function, \& they are increasingly discovered during Ultrasonography or Computed Tomography. However, in our study most of the patients were symptomatic $(77 \%) \&$ diagnosed on Ultrasound \& Computed Tomography (CT). Of the liver masses most common was hepatocellular carcinoma (61.6\%). Hepatitis-B infection ${ }^{3,4}$ and Hepatitis-C infection ${ }^{5}$ appear to be the most significant causes of hepatocellular carcinoma worldwide. This increase in proportion of hepatocellular carcinoma in our study was probably due to associated Hepatitis-B \& C infection. Hepatocellular carcinoma can co-exist with bile duct cancer or cholangiocarcinoma ${ }^{6}$. (16\%) cases in our study had co-existent bile duct cancer. 5\% of cirrhotic patients eventually develop hepatocellular carcinoma which is often multifocal. $(8 \%)$ in our study had cirrhosis.

Computed Tomography (CT), Delayed CT, Arterial portography with CT (CTAP), Magnetic Resonance Imaging (MRI), Ultrasound \& Intra-Operative Ultrasound (IOUS) all have been described as useful imaging techniques in patients being considered for hepatic resection ${ }^{1,2}$. While CTAP is very sensitive, it also has a relatively high false positive rate ${ }^{7}$. At many centers, IOUS \& CTAP have been found to be essentially equivalent in the ability to determine resectability of liver tumor ${ }^{8,9}$. These facilities were not 
available in our department, so our diagnosis \& management were solely on CT-Scan. Although CT can provide useful information about the segmental anatomy of the liver, there are no secure criteria for diagnosing hepatocellular carcinoma using CT although many observations are highly suggestive.

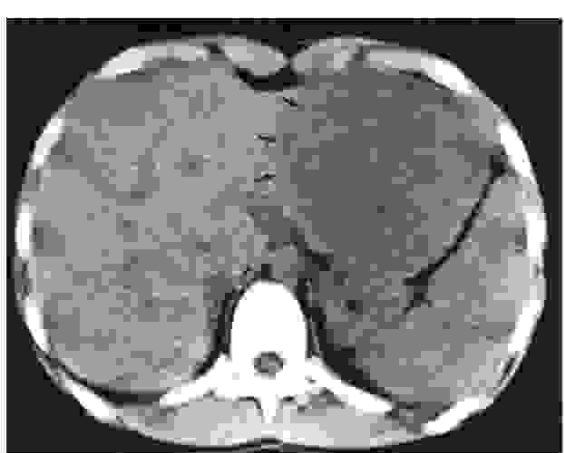

Control scan showing bypo dense area in the teft lobe As compared with the normal right lobe (good delineation of the tumour from the normal liver parenchyma).

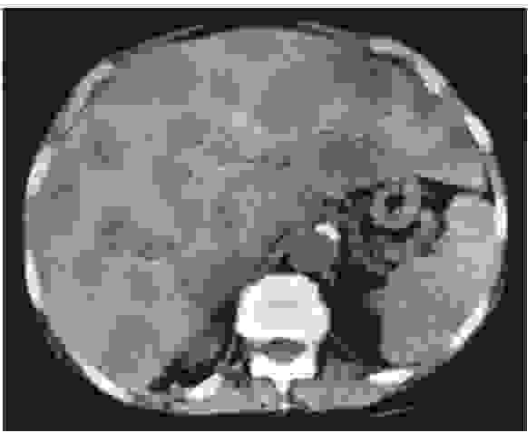

Plain scan:- multiple rounded hypodense lesiens within enlarged liver (Mets)

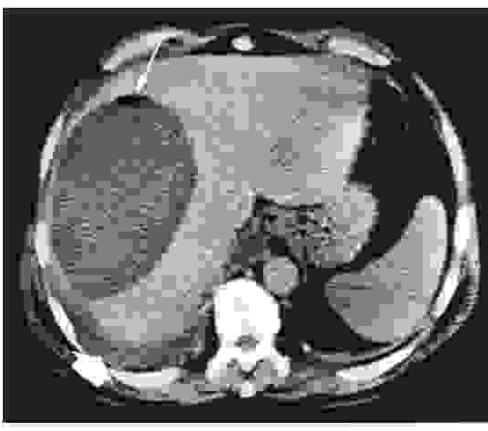

Pyogenic hepatic abscess.(control scan)

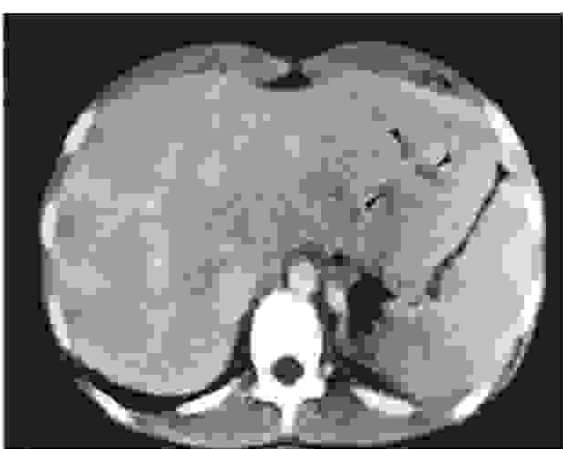

Decreased differential density between the tumour \& the normal hepatic tissues. Multiple a rounded hypo dense struotures with absence of any vascular branching within the lesion.

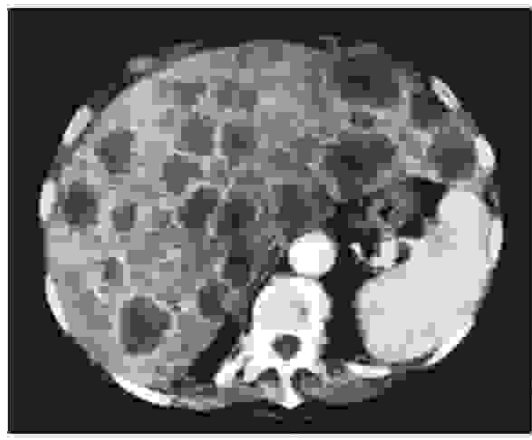

Contrast scan during the early arterial phase

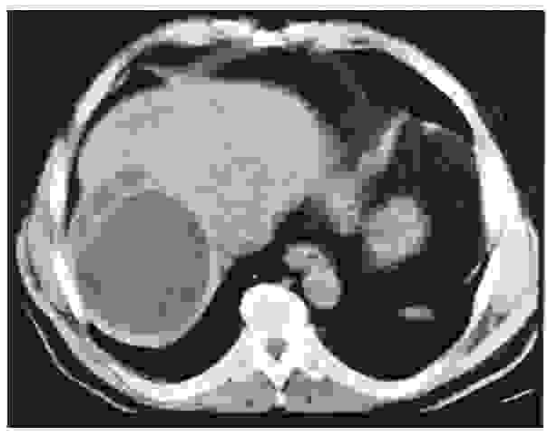

Hydatid cyst wilh daughter cysts (Control Scan)

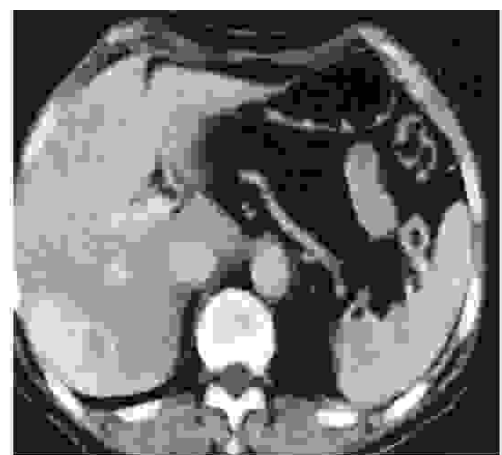

Post eontrast scan ( 2 minutes after hyper density of the lesion with attenution value close to intra hepatic blood vessels ( cavernous haemangioma)

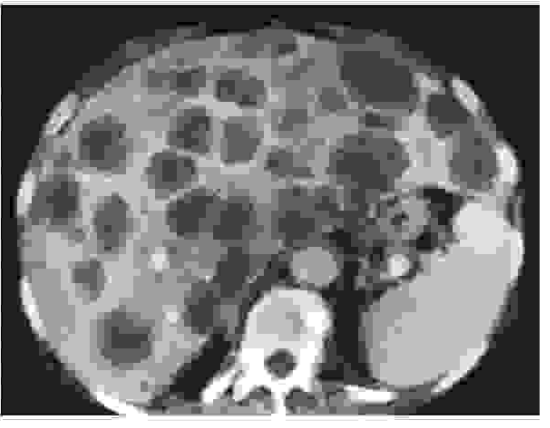

Contrast scan with enhancement of the hepatic parenchyna by the stained portal venous blood. The hvpodense mets are batter visible.

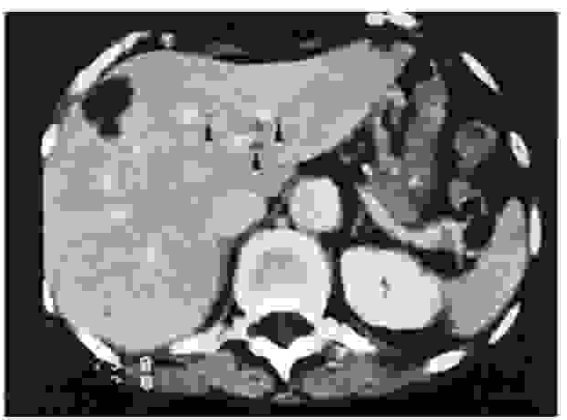

Post contrast scan benign hepatic cyst
A single expanding lesion or a few with attenuation close to that of normal hepatic parenchyma, or having a tendency to modify the liver outline, \& intense but non-uniform uptake of enhancement after bolus injection with rapid return to normal density are all characteristics to be considered critically when establishing the diagnosis of primary hepatic neoplasm. 
Modern imaging combined with recent technical advances in liver surgery can now offer many patients safe \& potentially curative resections for malignant \& benign conditions affecting the liver.

\section{REFERENCES}

1. Heiken JP, Weyman PJ, LEE JK, et al. Detection of focal hepatic masses: prospective evaluation with CT, delayed CT, CT during arterial portography, and MR imaging. Radiology 1989; 171:47-51.

2. Sitzman JV, Coleman J, Pitt HA, et al. Preoperative assessment of malignant hepatic tumors. Am J Surg, 1990;159:137-143

3. Mor E, Kaspa RT, Sheiner $P$, et al.: Treatment of hepatocellular carcinoma associated with cirrhosis in the era of liver transplantation. Ann Intern Med 129(8):643-53,1998.

4. Blumberg BS, Larouze B, London WT, et al.: The relation of infection with hepatitis $B$ agent to primary hepatic carcinoma. Am J Pathol 81 (3): 66982,1975
5. Tsukuma H, Hiyama $\mathrm{T}$, Tanaka $\mathrm{S}$, et al.: Risk factors for hepatocellular carcinoma among patients with chronic liver disease. N Engl J Med 328 (25): 1797801, 1993.

6. Jarnagin WR, Weber S, Tickoo SK, et al.: Combined hepatocellular and cholangiocarcinoma: demographic, clinical, and prognostic factors. Cancer 94(7): 2040-6, 2002.

7. Karl RC, Morse SS, Halpert RD, et al. Preoperative evaluation of patients for liver resection, appropriate CT imaging. Ann Surg. 1993;217:226-232.

8. Karl RC, Choi J, Yeatman TJ, et al. Role of computed tomographic arterial portography and intraoperative ultrasound in the evaluation of patients for resectability of hepatic lesions. J Gastrointest Surg. 1996.

9. Soyer P, Levesque M, Elias D, et al. Detection of liver metastases from colorectal cancer: comparison of intraoperative US and CT during arterial portography. Radiology. 1992;183:541-544.

\section{You must do the thing} you think you cannot do.

\section{Eleanor Roosevelt}

